This item was submitted to Loughborough's Research Repository by the author.

Items in Figshare are protected by copyright, with all rights reserved, unless otherwise indicated.

\title{
A novel re-initialization technique for CMA in the presence of channel noise
}

PLEASE CITE THE PUBLISHED VERSION

PUBLISHER

(C) IEEE

VERSION

VoR (Version of Record)

LICENCE

CC BY-NC-ND 4.0

\section{REPOSITORY RECORD}

Lambotharan, Sangarapillai, A.G. Constantinides, Jonathon Chambers, and K. Skowratananont. 2019. "A Novel Re-initialization Technique for CMA in the Presence of Channel Noise". figshare.

https://hdl.handle.net/2134/5814. 
This item was submitted to Loughborough's Institutional Repository (https://dspace.lboro.ac.uk/) by the author and is made available under the following Creative Commons Licence conditions.

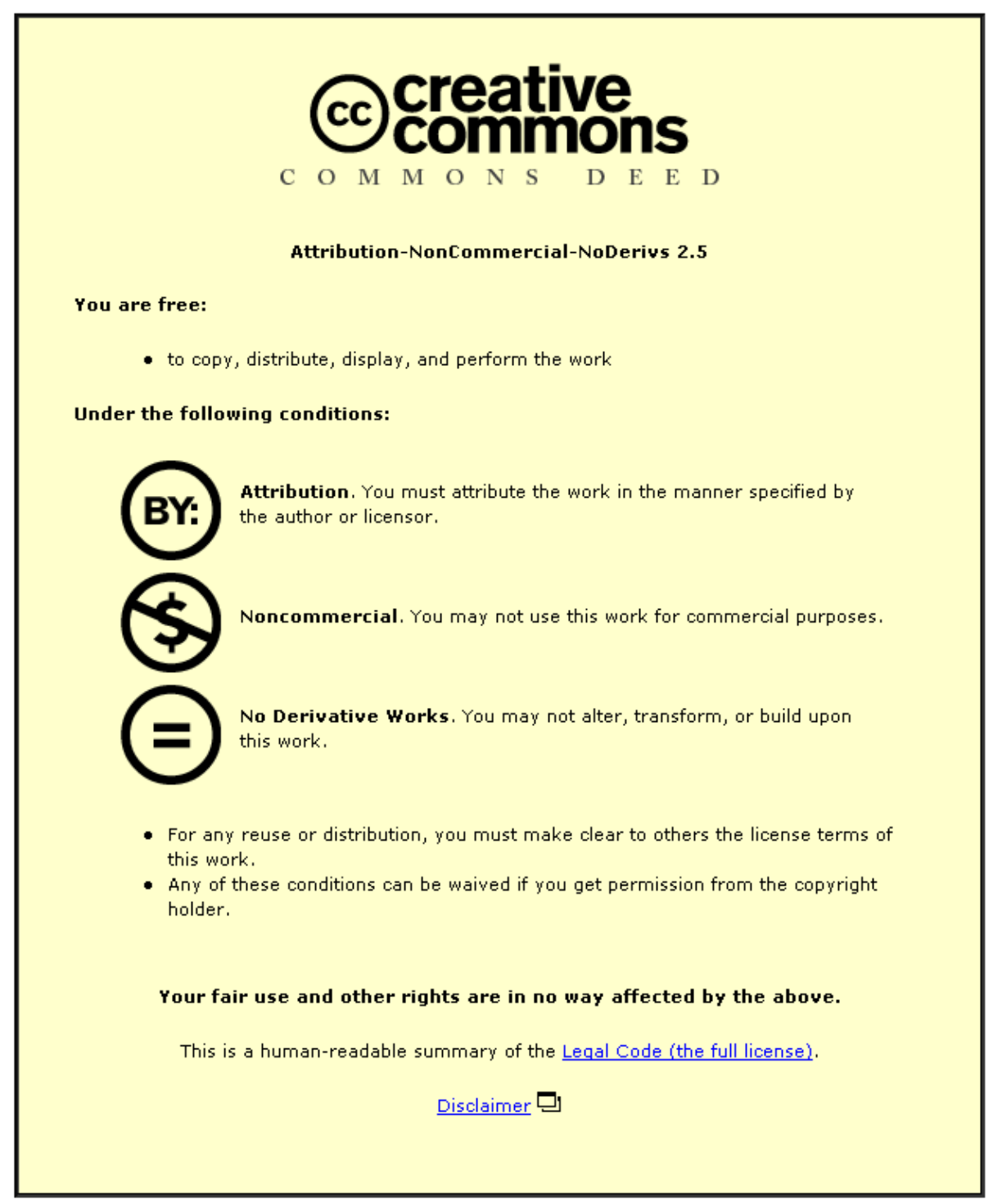

For the full text of this licence, please go to: http://creativecommons.org/licenses/by-nc-nd/2.5/ 


\title{
A NOVEL RE-INITIALIZATION TECHNIQUE FOR CMA IN THE PRESENCE OF CHANNEL NOISE
}

\author{
S. Lambotharan. A.G. Constantinides. I.A. Chambers and K. Shouratananont
}

Signal Processing and Digital Systems Section

Department of Electrical and Electronic Engineering

Imperial College. London, SW $\mathrm{S}$ 2BT

E-mail: s.lambotharanoic.ac.uk

\begin{abstract}
The error surface associated with the Constant Modulus Algorithm (CMA) is multimodal. Therefore, an equalizer adapted with CMA has the potential to converge to a local minimum that has large noise amplification. A technique is. therefore. proposed based upon a solution of linear and quadratic equations to re-initialise the equalizer parameters. Lincar equations are obtained from the fact that the retrieved transmitted signal with various delays which correspond to different minima are uncorrelated. The quadratic equation is obtained from the knowledge of the transmitted signal power. Simulations are included to demonstrate the robustness of this new scheme.
\end{abstract}

\section{INTRODUCTION}

The Fractionally Spaced Constant Modulus Algorithm (FS-CMA) does not posses local minima for an equalizer which models an inverse of a channel completely and in the absence of noise. All minima associated with this FS-CMA are of equal depth and they correspond to the retrieval of the transmitted signal with various delays and sign. However, when there is channel noise, as this noise is amplified by an amount which is equal to the squared $l_{2}$ norm of the equalizer impulse response, various minima behave differently and the minima with smallest equalizer norm are optimal. We provide a re-initialisation technique to obtain convergence to the global minima for CMA.

The first work in this context appeared in $[8]$. where a channel surfing and re-initialisation scheme was proposed. However, this method requires inversion of a matrix. Another method is a Gram-Schmidt orthogonalisation based adaptive algorithm for which a bank of at least two parallel equalizers is required [ $\bar{l}]$. A reference equalizer is adlapted with (MA while the second equaliser parameters are adapted according to a Gram-Schmidt orthogonalisation procedure, so that the second equaliser output does not have any contribution from the delaved transmitted signal that is being retrieved by the first equalizer. The CrossCorrelation and Constant Modulus. Algorithm (CC-CMA) that was proposed for the simultaneous retrieval of multi-user transmitted signals, [5] and [6]. was recommended for the optimum delay selection in [1]. A reference equalizer is adapted with the CMA algorithm and an another equalizer is adapted with the CC-CMA algorithm. The crosscorrelation term ensures that the second equalizer retrieves the transmitted signal with a particular delay that is different from the delay introduced by the reference equalizer. The first equalizer is initialised with the second one. if the second equalizer performs better than the first. The searching continues until the equalizer parameter with optimum delay is found. One other 
technique has been proposed based upon a fact that the equalizer parameter norm associated with the global minimum is small, and the following cost (for BPSK) is proposed to obtain global convergence, [2], [3] and [4],

$$
J=E\left\{\left(y^{2}(k)-1\right)^{2}\right\}+\kappa \mathrm{w}^{\mathrm{T}} \mathrm{w}
$$

where $\mathrm{w}$ is the equalizer impulse response vecfor and $f$ is a mixing parameter. The algorithm which aims to minimise (1) is a leaky constant modulus algorithm and this method cloes not require any searching procedure nor more than one equaliser. but the drawback is the bias in the position of the minima.

The technique introduced in this paper is a block based method to provide equalizers which retrieve the transmitted signal with various delavs which are different from the delay that is introduced by the reference equalizer. A matrix inversion as in [8] is required, but unlike as in [8], this scheme is also applicable to sub-cqualizers of length 1 . e.g. a beamformer.

\section{RE-INITIALISATION SCHEME}

The equalizer structure for the re-initialisation scheme is depicted in Figure 1. Let the impulse response vector of the channel, equalizer1 and equalizer-2 respectively be $\mathbf{c}, \mathbf{w}_{1}$ and $\mathrm{w}_{2}$. The equalizer-1 is adapted with CMA. Assume that the equalizer-1 retrieves the transmitted signal with delay $d$, i.e. $y_{1}(k)$ is approximately equal to $s(k-d)$. Let $\mathbf{x}_{\mathbf{k}}$ be the equalizer regressor vector. We desire to initialise equalizer-2 such that its output does not have any contribution from $s(k-d)$. Suppose we want $y_{2}(k)$ to be approximately equal to $s(k-d+\tau)$. then

$$
\begin{aligned}
& E\left\{y_{2}(k) y_{1}(k+i)\right\}= \begin{cases}0 & i \neq \tau \\
\sigma_{s}^{2} & i=\tau\end{cases} \\
& \mathbf{w}_{2}^{\mathrm{T}} \mathbf{R}_{\mathbf{i}} \mathbf{w}_{\mathbf{1}}= \begin{cases}0 & i \neq \tau \\
\sigma_{s}^{2} & i=\tau\end{cases}
\end{aligned}
$$

where $\mathbf{R}_{\mathbf{i}}=E\left\{\mathbf{x}_{\mathbf{k}} \mathbf{x}_{\mathbf{k}+\mathbf{i}}^{\mathrm{T}}\right\}$ and $\sigma_{s}^{2}$ is the power of the transmitted signal. For a channel of

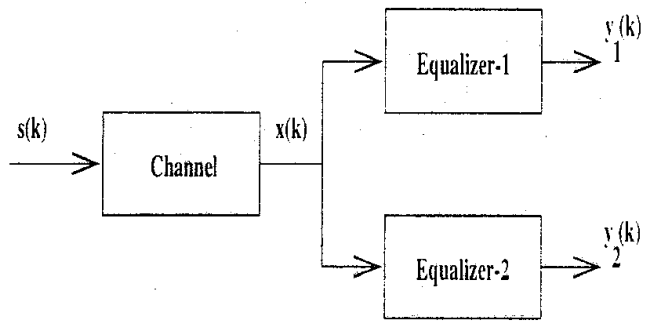

Figure 1: The equalizer structure for the reinitialisation scheme.

order $M$ and an equalizer of order $N .0 \leq$ $d \leq(M+N)$. Therefore, in order to consider' all possible delays, the range for $i$ should be $-(M+N) \leq i \leq(M+N)$. Write $\mathrm{R}_{\mathrm{i}} \mathrm{w}_{\mathbf{1}}$ $=\mathrm{u}_{\mathrm{i}}$ which is also equal to $E\left\{\mathrm{x}_{\mathrm{k}} y_{1}(k+i)\right\}$. From eqn.(3), we have $\mathbf{u}_{i}^{\mathbf{T}} \mathbf{w}_{\mathbf{2}}=0 . \quad i \neq \tau$ and $\mathrm{u}_{\mathrm{i}}^{\mathrm{T}} \mathrm{w}_{2}=\sigma_{s}^{2}, \quad i=\tau$. In addition to this set of equations, we have the power nomalisation which yields

$$
\mathbf{w}_{2}^{\mathrm{T}} \mathbf{R}_{0} \mathbf{w}_{2}=\sigma_{s}^{2}
$$

Solving eqn.(3) and eqn.(4) means fincling the intersection of a set of hyper-planes with a hyper-ellipsoid. Write $p=M+N$. U $=\left[\begin{array}{lllllll}\mathbf{u}_{-\mathrm{p}} & \cdots & \mathbf{u}_{\tau-1} & \mathbf{u}_{\tau} & \mathbf{u}_{\tau+1} & \cdots & \mathbf{u}_{\mathrm{p}}\end{array}\right]^{\mathrm{T}}$. The Least Square (LS) solution is

$$
\mathrm{w}_{2}=\left(\mathrm{U}^{\mathrm{T}} \mathrm{U}\right)^{-1} \mathrm{U}^{\mathrm{T}} \mathrm{e}_{\mathrm{v}}
$$

where $e_{v}$ denotes a vector with 1 in the is $v^{\text {th }}$ position and zeros elsewhere. c.g... $\mathrm{e}_{2}=$ $\left[\begin{array}{llllll}0 & 0 & 1 & 0 & \cdots & 0\end{array}\right]^{T}$ and $y-p=\tau$. (onsidering the power normalisation of equ.(4), $w_{2}$ is written as

$$
\mathrm{w}_{2}=\sqrt{\frac{\sigma_{s}^{2}}{\mathrm{w}_{2}^{\mathrm{T}} \mathrm{R}_{0} \mathrm{w}_{2}}} \mathrm{w}_{2}
$$

and the second equaliser is initialised at $\mathrm{w}_{2}$. The square error associated with the LS solution of $(5)$ is

$$
\sigma_{v}^{2}=1-\mathbf{e}_{\mathbf{v}}^{\mathbf{T}} \mathbf{U}\left(\mathbf{U}^{\mathbf{T}} \mathbf{U}\right)^{-1} \mathbf{U}^{\mathbf{T}} \mathbf{e}_{\mathbf{v}}
$$

Various initialisations are obtained for different values of $v$ except for $v=p$. Those initialisations such that $\sigma_{v}^{2}$ is approximately unity are discarded as $\sigma_{v}^{2} \approx 1$ indicates that the corresponding delay is outside the possible range of delay that the channel and the second equalizer can span, i.e. $d-\tau \notin(0 . M+N)$. 


\section{SIMLLATIONS}

Three simulations were performed to rerify the potential of this method. First a fracfonally spared chanel was simulated as subhanred $=0.940 .4-2$ and sub-channel$2=0.4+1-0.142$. The noise power at each semsor in 0.1. i.e. SNR $=10 \mathrm{~dB}$. There are two local minma and two globe ininima. The equalizer is initialised at $0.5-0.5$ and adapted with ('MA. and the equalizer converges to a local mininum where the Mean Square Error (MSE) is measured to be 0.215.). The equalizer is then re-initialised which is marked by $*$ (Figure 2 ). and this initialisation is very close to the global minimum where the MSE is measured to be 0.0699 .

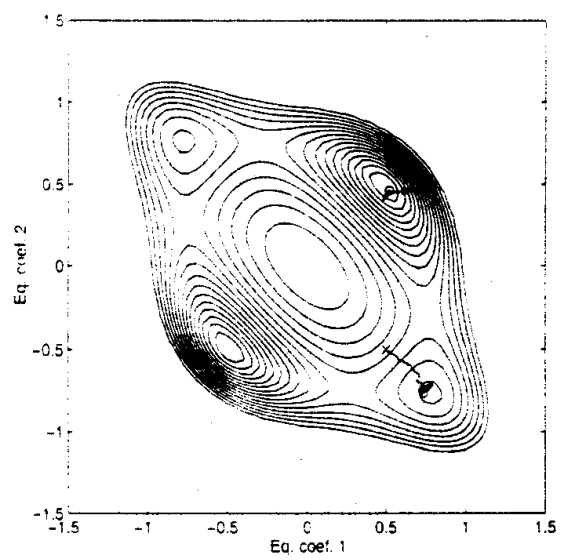

Figure 2: The contours associated with CMA, re-initialisation point. ${ }^{*}{ }^{*}$. and the convergence trajectories for a fractionally spaced equalizer.

The second example considers a baud spaced channel $\left[\begin{array}{ll}0.8944 & 0.4472\end{array}\right.$ and an equalizer of length 2. The equalizer is initialised at $\left[\begin{array}{ll}0 & 1\end{array}\right]$ and adapted with CMA. Convergence to a local minimum is observed. Figure 3 . SNR is 10 dB. An initialisation point which is marked by ${ }^{\cdot{ }^{*}}$ is obtained with our proposed method which leads CMA to a global minimum. The MSE at the local minimum and the global minimum are 0.2661 and 0.1759 .

The final example considers a fractionally spaced channel of length 4 . and an equalizer

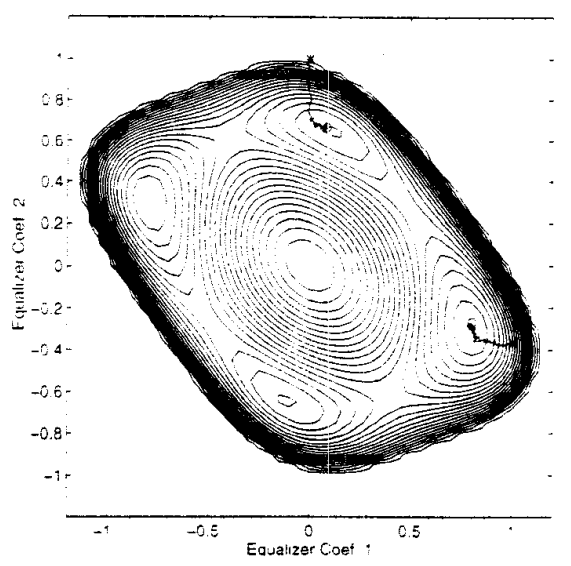

Figure 3: The contours associated with (MA. re-initialisation point. ${ }^{*}$. and the convergence trajectories for a baud spaced equalizer.

of length 3. Therefore. there are 6 possible delays. Suppose. we know the channel impulse response. a Minimum Mean Square Error (MMSE) equalizer for a desired delay $d$. can be designed as follows

$$
\mathbf{w}=\left(\mathbf{C}^{\mathrm{T}} \mathrm{C}+\frac{\sigma_{n}^{2}}{\sigma_{s}^{2}} \mathbf{I}\right)^{-1} \mathbf{C}^{\mathbf{T}} \mathbf{e}_{\mathrm{d}}
$$

where $\mathbf{C}$ is the channel convolution matrix. $\sigma_{s}^{2}$ and $\sigma_{n}^{2}$ are respectively the signal and the noise power. The MSE at this delay $d$ is given by

$$
\sigma_{\epsilon}^{2}=\sigma_{s}^{2}\left(1-\mathbf{e}_{\mathbf{d}}^{\mathbf{T}} \mathbf{C}\left(\mathbf{C}^{\mathbf{T}} \mathbf{C}+\frac{\sigma_{n}^{2}}{\sigma_{s}^{2}} \mathbf{I}\right)^{-1} \mathbf{C}^{\mathbf{T}} \mathbf{e}_{\mathbf{d}}\right)
$$

For a suls-channel- $1=0.7-5.20 .0642-0.2502-$ $0.5725]$ and sub-channel-2 $=[-0.14090 .7902$ $0.4599-0.3798$ ]. MSEs of the MMSE equalizers which correspond to 6 different delays were obtained. Figure 4. The optimum delay is 1 . The equalizer is initialised at $[-0.0374-$ $0.1951-1.0311 \quad 0.27170 .6906 \quad-0.3585]$ which is the MMSE equalizer that corresponds to the worst delay, i.e.. 5. and adapted with CMA. This equalizer converges to a local minimum where MSE is large (0.2923). The reinitialization parameters were obtained according to eqn.(5). Eqn.(7) indicates that the obtained initial CM solution is for delay 5 . and five other solutions can be obtained from the first five columns of $\left(\mathbf{U}^{\mathbf{T}} \mathbf{U}\right)^{-\mathbf{1}} \mathbf{U}^{\mathbf{T}}$. A bank 
of five parallel equalizers was initialized with these parameters, and adapted with CMA. The MSE at those minima are depicted in Figure 4. The MSEs at the global minimum is 0.1242 . A sequential search can also be performed instead of emploving these five parallel equalisers, and in this case, the parameters that correspond to the smallest element of $\sigma_{v}^{2}$ can be used as an initial guess.

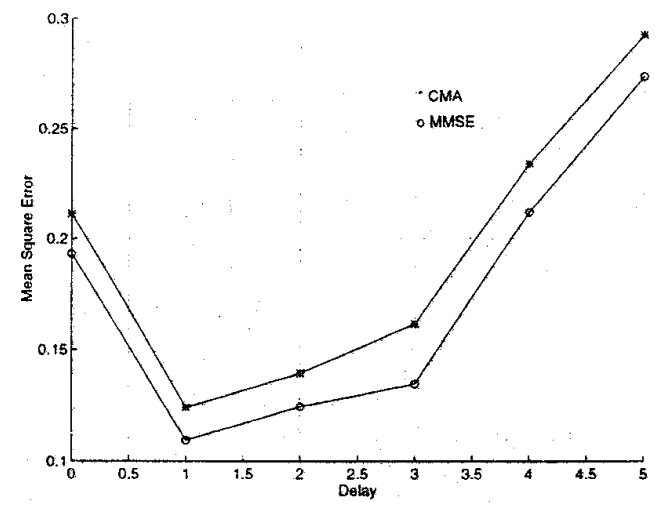

Figure 4: MSE of the MMSE equalizers and the CMA equalizers for various delays

\section{CONCLUSION}

We proposed a crosscorrelation based re-initialisation scheme for constant modulus algorithms to obtain convergence to a global minimum. This method involves estimation of the autocorrelation matrix of different delays, and performing an inverse of a matrix; hence, it has a computational complexity that is similar to [8], but provides re-initialization parameters for all possible delays simultaneously. Unlike [8], this scheme is also capable of providing solutions for FSE of length (sub-equalizer) one, i.e. a beam former. We demonstrated that these methods can be successfully applied to an FSE to obtain global convergence where the noise amplification is small. Moreover, this method can also be applied to obtain possible global convergence for a baud spaced equalizer.

\section{REFERENCES}

[1] D.J. Brooks, S. Lambotharan and J.A. Chambers. "Optinum Delay and Mean Square Error Ising (MA." ICASSP. Seattle, vol. 6. pp. 3361-3364. May 1998.

[2] S. Lambotharan, A.G. Constantinides and J.A. Chambers, "Channel Equalization Under Constant Modulus and Reduced Noise Amplification Objectives", IEEE DSP Workshop, Ftah. Aug. 1998.

[3] S. Lambotharan, A.G. Constantinides and J.A. Chambers, "Constant Modulus Receivers Under Reduced Noise Amplification Objective," submitted for $1 \mathrm{EE}$ Proc. on Communications, Feb. 1998.

[4] S. Lambotharan, J.A. Chambers and A.G. Constantinides , "Leaky Constant Modulus Algorithms: Sensitivity of Local Minima," submitted to IEEE Trans. Sig. Proc., Sept. 1998.

[5] S. Lambotharan, and J.A. (hambers, "On the Surface Characteristics of a Mixed Constant Modulus and CrossCorrelation for the Blind Equalisation of an MIMO Channel", accepted for Signal Processing, Sept. $199 \pi$.

[6] C.B. Papadias and A..]. Paulraj. A C'onstant Modulus Algorithm for Multiuser Signal Separation in Presence of Delay Spread Ising Antenna Arrays". IEEE Sig. Proc. Letters, vol. 4, 110. 6. p). 178181, June 1997.

[7] K. Skowratananont. S. Lambotharan and J.A. Chambers, "Improved Convergence of Fractionally Spaced Constant Modulus Equalizers in Presence of Noise." IEE Electronics Letter, vol. 34, no. 17. 16441646, Aug. 1998.

[8] L. Tong and H.H. Zeng, "(thamelSurfing Re-initialization of CMA“. IEEE Workshop in Signal Processing Advances in Wireless Communications SPAWC, Paris, pp. 45-48, 1997. 